\title{
Two months old with arachnoid cyst and unusual clinical presentation
}

\author{
Tomas Munoz, Joanne Holliman, S. Khanna*
}

Division of Neonatology, Department of Pediatrics, Lincoln Medical and Mental Hospital, New York, USA;

*Corresponding Author: sureshkhanna89@gmail.com

Received 11 April 2013; revised 12 May 2013; accepted 17 June 2013

Copyright (C) 2013 Tomas Munoz et al. This is an open access article distributed under the Creative Commons Attribution License, which permits unrestricted use, distribution, and reproduction in any medium, provided the original work is properly cited.

\begin{abstract}
Arachnoid cysts are relatively uncommon in infancy and are incidental findings in intracranial imaging in pediatric patients. Presentation at a younger age is usually associated with cyst enlargement. Arachnoid cysts are considered to be congenital in origin but the natural history and prevalence is not well defined. Here we report a case of a 2 months old male infant with nonspecific illness who was diagnosed with arachnoid cyst.
\end{abstract}

Keywords: Arachnoid Cyst

\section{CASE REPORT}

A 2 months old male infant was brought to Emergency Department (ED) with mother complaining of "not acting as usual" for 5 days. According to the mother, the infant was sleepy, very fussy and feeding less than before. He was voiding well and having regular bowel movements. The infant is also reported to have mild runny nose for 2 days prior to ED visit. Infant was seen by his regular pediatrician 4 days prior to the ED visit with a history of excessive crying and was discharged with a diagnosis of "Colic".

In the ED the infant was afebrile, with a weak cry and rhinorrhea accompanied by mild pharyngeal erythema and prolonged expiratory wheezing. Blood samples were sent for cultures and complete white blood cell count (CBC), chemistry and a urine sample was sent for analysis and cultures. Pharyngeal secretions for viral panel. Chest roentgenogram (CxR) was done and reported to be normal except for a questionable fracture of 10th Rib.

The physical exam showed that infant was a was well nourished and hydrated, "sleepy" but arousable with fair to good suck, generalized hypotonia and irritability and decreased movement of upper extremities with normal reflexes. There was no fasciculation of the tongue and the eye exam was normal. The remainder of the physical examination was within defined limits. Skeleton survey was normal (child abuse was ruled out). CT scan of brain without contrast was normal.

Infant was admitted to Pediatric intensive care unit (PICU) for further treatment and a spinal tap was performed and the infant was started on antibiotics. The laboratory results were as follows: Complete Blood count (CBC): white cell count 9700, hemoglobin-12.1 gm/dl, Platelet count-597k, differential count was normal. Serum electrolytes: sodium 137, potassium 5.6, chloride 106 , bicarbonate 23 , blood urea nitrogen 5 , serum creatinine $<0.5$, calcium 9.8, magnesium 1.8 and phosphorus 5.8. Liver function tests were normal, urine analysis was negative for protein, glucose, leukocyte esterase and reducing substance, the viral panel was negative and blood and urine cultures were negative. Cerebrospinal fluid analysis and cultures were normal. Blood ammonia was $26 \mathrm{Umol} / \mathrm{L}$, arterial blood gas-pH: 7.401, $\mathrm{PCO}_{2}$ 42.0, $\mathrm{PO}_{2}$ 90, $\mathrm{TCO}_{2}$ 27, $\mathrm{HCO}_{3}$ 26.1, and BE 1 .

Vital signs: Temperature-99.7F; heart rate 150/minute; respiratory rate $38 /$ minute; blood pressure $87 / 43 \mathrm{~mm} \mathrm{Hg}$ and oxygen saturations; $99 \%$ on room air. Wt: $4.6 \mathrm{~kg}$ (25\% tile), HC: $40 \mathrm{~cm}$ ( $40 \%$ tile).

Infant's growth and development were normal and all immunizations were up to date. Family history was non contributory, had a 5 years old sibling in good health.

Birth history: Full term infant was delivered by caesarean section for Category II tracings. Apgar score was 9 and 9 at 1 and 5 minutes respectively. Infant's birth weight was $3025 \mathrm{~g}$ and head circumference $35 \mathrm{~cm}$. Infant's newborn course was uneventful and was discharged home with his mother on 3rd day of life.

In the PICU infant's examination revealed that the infant was a well nourished and sleepy with intermittent shallow respiration, weak cry on stimulation, decreased activity and hypotonia. The infant appeared uncomfortable when held from the trunk and had hyperesthe- 
sia .The rest of the physical examination was significant for a brief episode of tachycardia in $180 \mathrm{~s}$ (electrocardiogram showed sinus tachycardia) at the time of admission.

While in PICU, the infant became very lethargic while being held in mother's lap, with shallow respiration, bradycardia, cyanosis and oxygen saturations in the $70 \mathrm{~s}$ $\left(\mathrm{SpO}_{2}\right)$ The infant subsequently developed apnea and was unresponsive and was immediately intubated and placed on a ventilator. He was unresponsive during the procedure.

The infant was transferred to a tertiary care center for further management. Magnatic Resonance imaging( MRI) of brain with and without contrast showed fluid a filled cyst consistent with an arachnoid cyst measuring $1.7 \mathrm{~cm}$, compressed upper cervical spinal cord below the foramen magnum. No Chiari malformation was detected. The ventricles were normal in caliber, without hydrocephalus. There was no evidence of hemorrhage or hemosiderin deposition within the brain (Figures 1 and 2).

The cyst was decompressed with a C1-C2 laminectomy and the postoperative course was uneventful. Infant

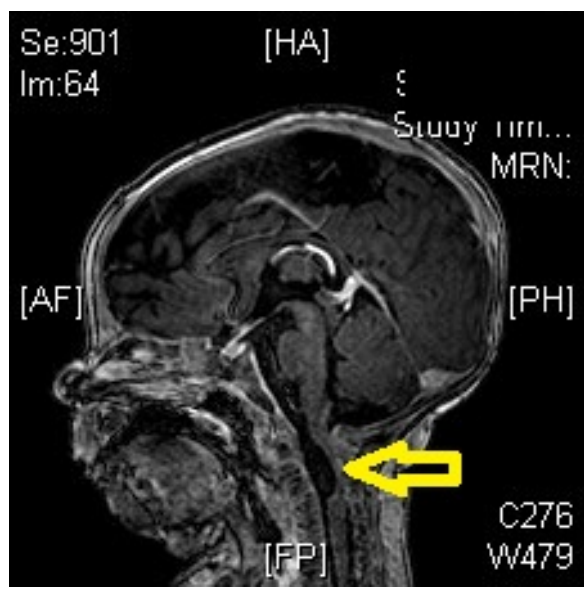

Figure 1. MRI brain 1.

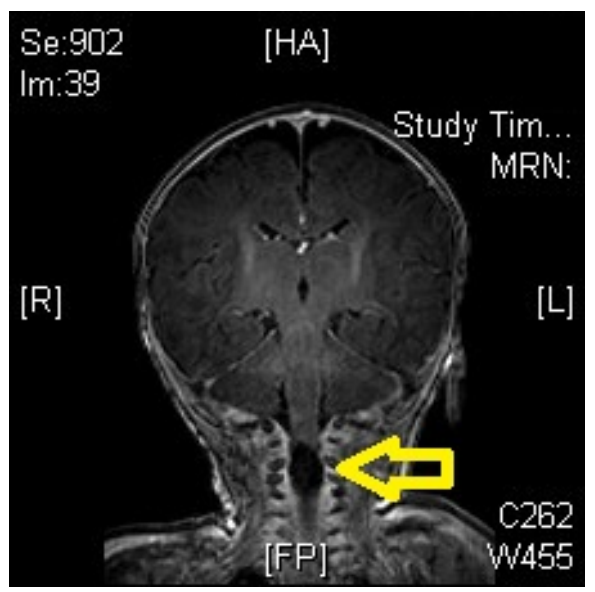

Figure 2. MRI brain 2. was feeding well and had no more episodes of apnea, bradycardia or weakness. The arachnoid cyst fluid analysis showed bloody fluid with WBC 75, RBC 57500, Lymphocytes 66, Glucose $8 \mathrm{mg} / \mathrm{dl}$, Protein $<0.3$.

At the time of discharge, the results of infant's physical examination were age appropriate and on subsequent clinic follow up appointments.

\section{DISCUSSION}

Since the earliest known description by Brigal et al. in 1831 , there have been numerous case reports of arachnoid cysts. These are rare lesions that accounts for $<1 \%$ of all intracranial masses [1]. They are benign, fluid filled cysts that contains fluid similar to cerebrospinal fluid without any genetic association [2]. Arachnoid cysts can be congenital (most common) or secondary in nature. Congenital cysts are considered developmental anomalies that results from splitting or duplication of the arachnoid membrane during early embryological life. The acquired or secondary type of arachnoid cysts contains either fluid that stains positively for hemosiderin with or without inflammatory cells [1].

The exact incidence of arachnoid cysts and natural history in children are unknown, the majority of the arachnoid cysts are detected incidentally findings on a CT scan or MRI performed for other reasons. The majorities of these cysts are found in the middle fossa, with left sided cyst occurring more commonly than right sided cysts and are preponderant in males [3]. Most of these cysts are static in nature although some involutes and disappear completely. The average age at the time of presentation was 4 yrs 7 months (1 day - 1 year) [2].

Associated anomalies are rare and the most common being hydrocephalus. There have been a few reported cases of concurrent some micro-opthalmia or achondroplasia.

Clinical symptoms of arachnoid cysts are usually nonspecific and depend on the size and location of the cysts and impact on CSF flow. Small cysts are usually asymptomatic. The most common symptoms are related to increased on area the surrounding the cyst. Seizures, craniomegaly, developmental delays, headache, gait disturbances, vomiting and weakness are commonly reported. $[1,3,4]$

As most of these cysts are static in nature, conservative management is recommended in most cases with a close follow up with a CT scan or MRI. Since arachnoid cysts are congenital in origin, antenatal diagnosis is possible due to widespread use of antenatal ultrasound. There is no consensus on management of these patients. Some authors believe that even the asymptomatic cysts should be removed to avoid the potential risks of compression on surrounding structures or rupture. The two most common removal procedures are cystoperitoneal 
shunting or fenestration. Complete cysts excision is not possible and the best approach is still controversial [5].

Our patient was only 2 months old at time of presentation and had vague symptoms of sleepiness, irritability, hypotonia, hyperesthesia, poor feeding, apnea, bradycardia and desaturations, can be due to arachnoid cysts compressing the cardio-respiratory centers located in brainstem area.

\section{CONCLUSION}

Arachnoid cysts are benign congenital lesions containing of CSF like fluids and are usually identified upon examination of CT scans or MRI performed for unrelated reasons. Clinical symptoms are usually non-specific but depend on the size and location of cysts. Management of theses lesions is still controversial.

\section{REFERENCES}

[1] Cincu, R., Agrawal, A. and Eiras, J. (2007) Intracranial arachnoid cysts: Current concepts and treatment alternatives. Clinical Neurology and Neurosurgery, 109, 837843. doi:10.1016/j.clineuro.2007.07.013

[2] Wang, P.-J., Lin, H.-C., Liu, H.-M., Tseng, C.-L. and Shen, Y.-Z. (Year) Intracranial arachnoid cysts in children: Related signs and symptoms. Pediatric Neurology, 19, 100-104.

[3] Al-Holou, W.N., Yew, A.Y., Boomsaad, Z.E., Garton, H.J., Muraszko, K.M. and Maher, C.O. (2010) Prevalence and natural history of arachnoid cysts in children. Journal of Neurosurgery. Pediatrics, 5, 578-585.

[4] Erdincëler, P., Kaynar, M.Y., Bozkus, H. and Cëiplak, N. (1999) Posterior fossa arachnoid cysts. British Journal of Neurosurgery, 13, 10-17.

[5] Jayaprakash, A. and Gosalakkal, M.D. (2002) Intracranial arachnoid cysts in children: A review of pathogenesis, clinical features, and management. Pediatric Neurology, 26, 93-97. 